\section{REPORT FROM THE ADHA}

\section{Ailsa Colquhoun attended the recent American Dental Hygienists Association conference in Albuquerque, New Mexico and sent \\ Vital the following report.}

\section{Tobacco cessation}

Dental surgery staff have good interviewing, educating, motivating and counselling skills - so why don't dental surgeries get more involved in public health? Smokers should be a particular target, as most will try an average of between three and eight times before they succeed in a quit attempt, tobacco cessation specialist and dental hygienist Carol Southard told delegates,

in a conference presentation aimed at dental hygienists. Carol believes that the single most

helpful intervention in a quit attempt is to understand a smoker's quit 'state'. For example, if the smoker is not ready to consider quitting, the aim should be to put a quit attempt on the agenda within the next six months. A discussion about how the smoker feels about quitting can then be facilitated by reviewing the advantages of quitting versus the inconveniences and risks of smoking.

If the smoker is planning to quit within six months but has not set a stop date, the conversation should aim to reassure the smoker that the advantages of quitting will be more significant than the inconveniences. 'You may need to identify the obstacles and explore solutions,' advises Ms Southard, 'or encourage the smoker to picture life as an ex smoker. In both cases, you can employ the 'teachable moment', that is to link the patient's oral health condition with a talk about smoking cessation.'

\section{Obesity}

Reducing obesity rates is also an area for dental team input. In England, nearly a quarter of adults and about
$10 \%$ of children are obese. Obesity is linked to heart disease, cancer, diabetes, but also to periodontal disease.

Obese patients are in a chronic state of low grade inflammation. Adipokines, which are released in proportion to the mass of adipose tissue, contribute to the continual activation of the immune response. It is this immune response that is considered to be a primary cause of bone loss in periodontal disease.

As well as educating patients on the dental risks associated with obesity, dental care staff can signpost patients to the GP for help with weight loss. They can also cater for the obese/ overweight patient by providing practice equipment that does not have a weight bias, said researcher Kristen Karns and colleagues from the Clark College of Dental Hygiene, Vancouver, Washington, in their presentation 'Obesity. A dental concern'.

\section{Catering for special need patients}

Making patients feel comfortable during their visit is key to maintaining regular contact. In the USA, dental phobia is a key treatment compliance issue, with half of the population afraid of the dentist. Around 30 million Americans avoid going to the dentist altogether.

Dental hygiene treatment evokes more fear than dental treatment, researchers from the New York University College of Dentistry believe, with patients reporting the highest pain ratings from probing and manual/ ultrasonic cleaning. This is why

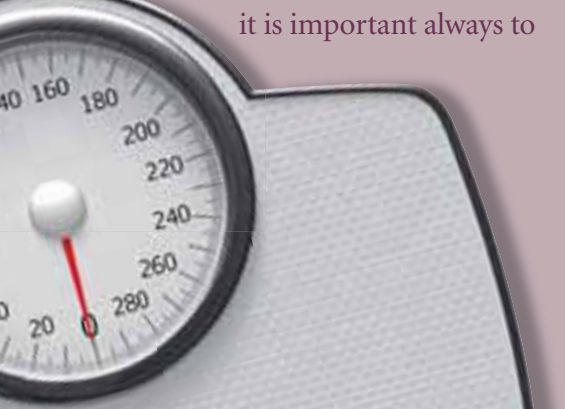

meet and greet your patient, to explain the treatment plan in layman's terms and to have good justification for your procedures, said researchers Stephanie Kim and Vincent Wong.

The same approach holds for patients with special needs, for example, deafness, added undergraduates from the school of nursing and dental hygiene at the University of Hawaii. As well as learning the sign language for terms such as exam, $\mathrm{x}$-ray, clean or brush teeth, dental care staff can help patients though simple measures such as:

- Removing their masks while speaking

- Maintaining eye contact even where there is an interpreter

- Using visuals (including a pain scale for assessing pain).

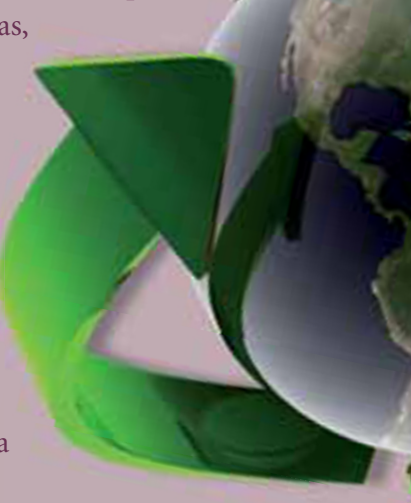

\section{Go green and save money}

Offices making energy

savings for the first time can save about $10-20 \%$ on energy bills with very little effort - and can make a positive contribution to the environment, according to Katie Clark and colleagues from The Ohio State University of Dentistry and Dental Hygiene.

In the USA, the healthcare sector is the number four contributor of mercury to the environment. Dentistry alone contributes $1 \%$ of the nation's total mercury waste.

To help the environment, why not consider going green by using:

- Cloth patient bibs and head rest covers

- Recycled paper products

- Toothbrushes made from recycled materials

- Energy saving washers and dryers

- Biodegradable paper cups

- Eco-friendly soaps and cleaning solutions

- Fluorescent lighting. 